\title{
Deprescribing in palliative patients with cancer: a concise review of tools and guidelines
}

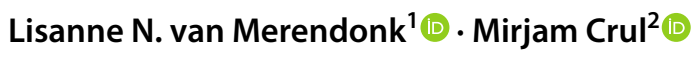

Received: 6 July 2021 / Accepted: 28 September 2021 / Published online: 7 October 2021

(C) The Author(s) 2021

\begin{abstract}
Purpose Palliative cancer patients can benefit from deprescribing of potentially inappropriate medications (PIMs). Tools and guidelines developed for the geriatric population are mainly available. This systematic review gives an overview of available guidelines and tools to deprescribe for palliative cancer patients.

Methods A systematic search was carried out using the databases SCOPUS and PubMed. Studies focused on palliative cancer patients were included.

Results The search identified 137 studies of which 15 studies were included in this systematic review. Six of the included tools were developed specifically for cancer patients. One of these tools was externally validated and applied in several studies and settings. Guidelines or tools that were not specifically developed for cancer patients but that were applied on cohorts of palliative cancer patients were also included.

Conclusion Tools developed for geriatric patients contain drugs that are not inappropriate when used in the palliative cancer care setting. Tools developed for cancer patients are more suitable and can be applied in combination with stepwise methods to individualize deprescribing per patient. The tools and guidelines described in this systematic review can be used to further implement deprescribing in the clinical routine for palliative cancer patients.
\end{abstract}

Keywords Cancer $\cdot$ Deprescribing $\cdot$ Palliative care $\cdot$ PIMs

\section{Introduction}

The use of a multitude of drugs is often termed "polypharmacy." In general, most practitioners understand this term in a negative connotation, when patients are taking a relatively large number of different drugs, and/or a number of drugs for which the appropriateness may be questionable (potentially inappropriate medications (PIMs)). Polypharmacy has been associated with an increased risk for adverse events, a higher symptom burden, and a lower quality of life $[1,2]$. Especially patients with cancer can be prescribed many drugs: systemic anti-cancer treatments,

Lisanne N. van Merendonk

l.v.merendonk@nki.nl

1 Department of Pharmacy \& Pharmacology, The Netherlands Cancer Institute-Antoni Van Leeuwenhoek, Plesmanlaan 121, 1066 CX Amsterdam, The Netherlands

2 Department of Clinical Pharmacy and Pharmacology, Amsterdam UMC, VU Medical Center, Amsterdam, The Netherlands often combined with supportive drugs, and additional treatments to decrease symptom burden or alleviate side effects. Since cancer is a disease of which the incidence increases with age, many patients also suffer from one or more comorbidities for which drugs are in use. More often than not, this results in complex drug schemes that patients or their caregivers have to manage. Patients with cancer may transition at some point from being on a treatment with curative intent to palliative care. This transition is often accompanied by a limited life expectancy. At this point, the benefit-risk ratio of medications can shift, for example, in the case of drugs prescribed as primary prophylaxis. The occurrence of polypharmacy and the use of potentially inappropriate medications (PIMs) in patients with advanced cancer have been studied and described in several publications. The largest study researched a patient cohort from Sweden, including $>150,000$ individuals, and found that in the last month of life $60 \%$ of patients were on continued antihypertensive treatment, $17 \%$ on lipid-lowering drugs, and $19 \%$ on mineral supplements [3]. A review from 2014 identified 9 trials that examined the use of PIMs in palliative cancer patients, 
yielding percentages of patients that receive one or more PIMs from 22 up to $95 \%$, depending on the study population as well as on the criteria used to determine which drugs can be regarded as PIMs [4].

The concept of deprescribing, to reduce polypharmacy and/or the number of PIMs in patients, was defined by Scott et al. as: "a systematic process of identifying and discontinuing drugs in instances in which existing or potential harms outweigh existing or potential benefits within the context of an individual patients' goals, current level of functioning, life expectancy, values and preferences" [1]. This concept serves multiple aims, for it has been shown that reducing the pill burden of patients with a limited life expectancy can increase quality of life [2,5], decrease the risk of side effects or worse clinical outcome [6,7], and reduce healthcare costs $[3,8,9]$.

In conclusion, the benefits of deprescribing in patients with cancer in the palliative phase are well established. However, there is as yet no gold standard or consensus on a guideline or deprescribing tool to do so. In this review, we will summarize and compare the available options that have been published in the scientific literature.

\section{Methods}

\section{Inclusion and exclusion criteria}

We included studies when they included palliative cancer patients. Therefore, studies focusing on a population without palliative cancer patients were excluded. Studies applying a tool or certain criteria for screening of medications were included if they were applied to palliative cancer patients. All publications that included tools in palliative care settings, including those specific for cancer or not specifically developed for cancer patients, were eligible for inclusion. Studies not applying a tool or guideline were excluded. Studies focusing on one specific medication category were also excluded. Only electronic articles available in English were included.

\section{Search strategy}

We carried out a literature search in December 2020 using the databases SCOPUS and PubMed. We searched these databases with the following terms: (pallia* OR palliative care) AND (oncology OR oncol* OR cancer OR metastat*) AND (deprescribing OR deprescribe OR deprescription OR "de-prescribing" OR "inappropriate prescribing" OR "inappropriate medications" OR "inappropriate medication" OR "unnecessary prescription" OR "unnecessary prescriptions"). One author (LM) carried out the search.

\section{Data processing}

Two authors (LM, MC) independently screened titles and abstracts of studies retrieved by the literature search after removal of duplicates for eligibility. Afterwards, the full-text articles were assessed for inclusion by two authors (LM, $\mathrm{MC})$, also independently. When the decision on inclusion yes or no differed between the two authors, an ultimate decision was reached after discussing the content of the paper. Data extraction was conducted of the included studies and summarized.

\section{Results}

The search identified 137 studies of which 17 studies were eligible for inclusion (Fig. 1). There were no discrepancies between the individual scores of the two screening pharmacists. Eight studies applied a tool not developed for the oncology population or a palliative cancer care population. In two articles, the authors recommended a methodology specific for oncology patients without applying or validating the tool. Since a few studies applied the same tool, in total, nine different tools or guidelines were identified. Table 1 summarizes these tools and guidelines and Table 2 shows the outcomes of the studies applying these tools. All identified tools and guidelines are further described below.

\section{Tools specifically for cancer patients}

\section{OncPal}

Three of the included studies evaluated the use of the OncPal guideline specifically developed for deprescribing in palliative cancer patients. Lindsay et al. [10] describes the development and validation of this guideline against an expert opinion panel in a single-center study. The OncPal guideline was shown to match the deprescription of 617 medicines in 61 patients with an accuracy of $94 \%$ when compared to the expert panel. In the 61 patients, $70 \%$ were taking at least one PIM, and of the total medicines that were used in the patient group, $21.4 \%$ were assessed as PIMs. The OncPal guideline gives deprescribing advice on 8 classes of drugs. Two other studies also assessed PIMs using the OncPal method (Table 2). Marin et al. [20] compared PIMs prior to and after a palliative care consult in non-curative in patients with cancer. They expanded the medication categories in the OncPal with anticoagulants and benzodiazepine receptor agonists and showed that a palliative care consult could reduce the percentage of PIMs from 21 to $14 \%$ in palliative cancer patients. Wenedy 
Fig. 1 Preferred reporting item for systematic reviews and meta-analysis (PRISMA) flow diagram

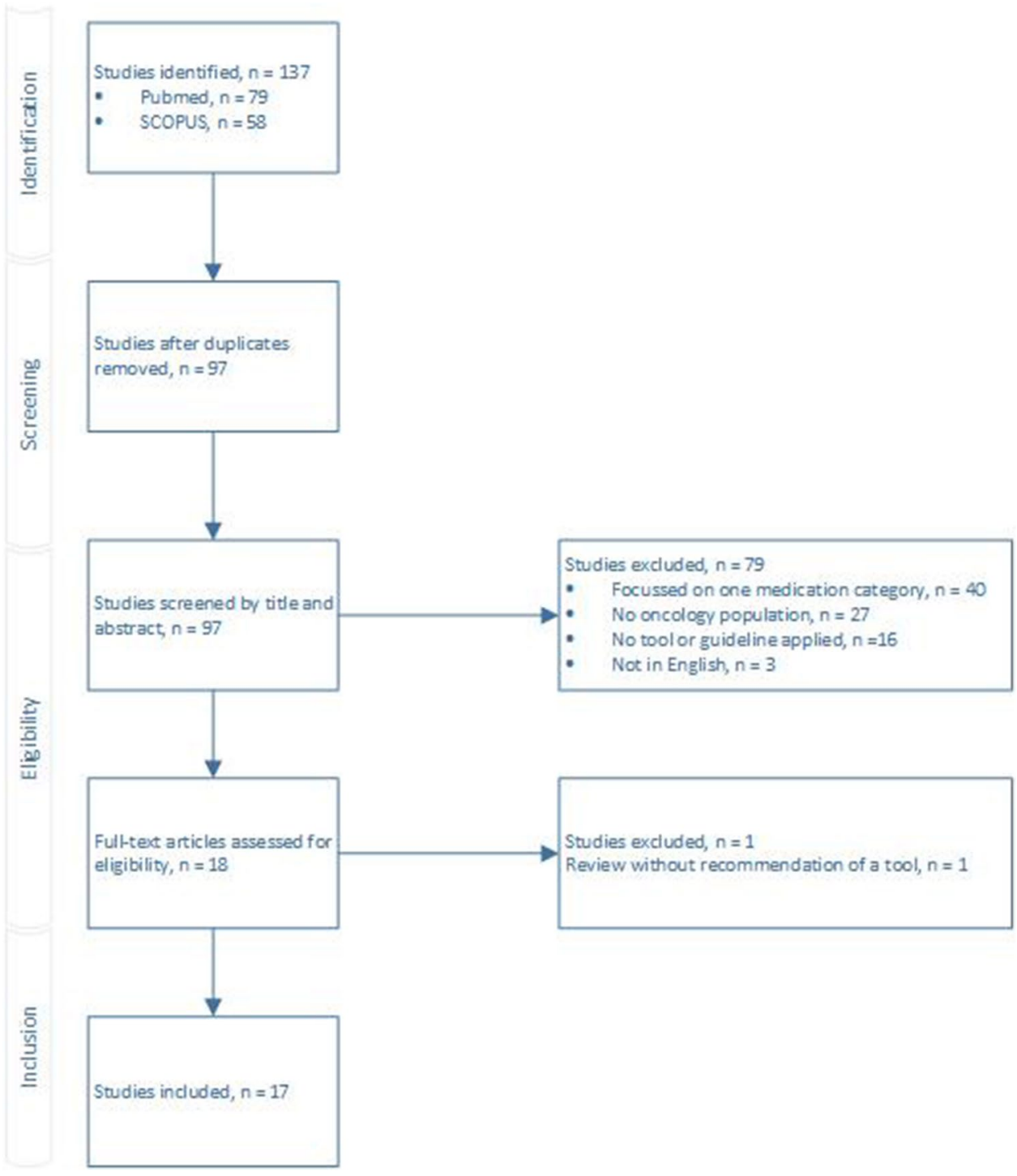

et al. [21] assessed the appropriateness of discontinuation of medications in cancer and non-cancer home hospice care patients using OncPal. No absolute amounts of PIMs were measured. However, the use of most of the preventive medications was discontinued in 60 to $70 \%$ of the included patients, with proton pump inhibitors being the drug class most often de-escalated or halted.

\section{6-Step method}

The 6-step method as proposed by Gonçalves [11] is described in Table 1. This is a systematic method to make deprescribing more efficient and safer. This method was suggested and described in a review. However, it gives no detailed guidance on which drugs for which indications can be safely deprescribed, nor has it been assessed in actual patients.

\section{Steps to deprescribe}

Also Sharma et al. [12] propose a stepwise method to deprescribe in older patients with cancer to increase appropriateness and safety during deprescribing (Table 1). These steps should be carried out periodically. This method was recommended for older patients with cancer and did not focus specifically on palliative cancer patients. Furthermore, like the 6-step method described above, this method has not been applied on a patient population.

\section{Futility criteria}

Oliveira et al. [13] modified criteria for futility from Fede et al. [14] into a guideline to assess futility with 7 medication categories (Table 1). These criteria were retrospectively applied on data of 448 advanced cancer patients referred to the palliative care service. These patients had a median survival of only 
Table 1 Summary of tools and guidelines identified

Tool Description

OncPal [10]

6-Step method [11]

Steps to deprescribe [12]

Futility criteria by Oliveira et al. [13]

Preventative medications by Todd et al. [15]

Medications for chronic diseases by Garfinkel et al. [16]

Beers criteria [17]
Validated against an expert opinion panel in a single-center study. It includes medications with a limited benefit in palliative cancer patients. It consists of 8 medication classes: anticoagulants, cardiovascular agents, osteoporosis medications, peptic ulcer prophylaxis, oral hypoglycemics, vitamins, minerals, and complementary-alternative medicines

A systematic method for deprescribing consisting of 6 steps

Step 0: reappraisal of the patient's clinical situation, setting treatment goals

Step 1: to find out all the medications a patient is taking

Step 2: agreement with patient and carers

Step 3: identify drugs that can be deprescribed in the first place without causing harm

Step 4: address medication that requires a long time until benefit, outside of the patients' expected lifespan

Step 5: identification of medications that could be withdrawn, but slowly

Step 6: monitor carefully to identify clinical problems

A periodically carried out comprehensive medication assessment following 5 steps to deprescribe:

Step 1: reconcile all medications and consider indications

Step 2: consider overall risk of harm

Step 3: assess each drugs in terms of current or future benefit in relation to current or future harm

Step 4: prioritize drugs for deprescribing, giving preference to those that have the most unfavorable risk/benefit ratio and least likelihood of withdrawal symptoms

Step 5: implement a discontinuation plan and monitor

Criteria for futility of 7 medication categories, criteria modified from Fede et al. [14]. Medication categories included conditions for futility. Medication categories covered gastric protectors, antihypertensive drugs, antidiabetic drugs, statins, anticoagulants, bisphosphonates, and antidementia drugs

Classes of the most common inappropriate preventative medication in patients with life-limiting illness based on a systematic review: vitamins and minerals, antidiabetic, antihypertensive, antihyperlipidemic, and antiplatelet medications

Medications for chronic diseases. Topical preparations and drugs for oncological treatments were excluded (oral and/or intravenous cytostatic drugs and biological agents)

PIMs to avoid by older adults in most circumstances or under specific situations, updated by the American Geriatrics Society
Target population during development

Palliative cancer patients with a life expectancy $<6$ months

Advanced cancer patients

Older patients with cancer

Advanced cancer patients with a life expectancy $<6$ months

Patients with a life-limiting illness

End-stage cancer patients referred to homecare hospice

Geriatric population 
Table 1 (continued)

\begin{tabular}{lll}
\hline Tool & Description & Target population during development \\
\hline STOPP criteria [18] & $\begin{array}{l}\text { Screening tool of older people's prescription } \\
\text { (STOPP) criteria consists of } 80 \text { criteria. }\end{array}$ & Older patients \\
& These medications are associated with \\
& adverse drug events and can be used for \\
& older people \\
& A questionnaire of 10 questions used by physi- & Ambulatory, elderly patients \\
Medication appropriateness index [19] & cians to fill in a score to assess if the use of a \\
& certain drug is appropriate of inappropriate. \\
& Questions are focused on, e.g., indications, \\
& dosage, durations, interactions, and effective- \\
& ness \\
\hline
\end{tabular}

15 days. The authors noted very high numbers of patients that were still on gastric protectants (50\%) and statins (97\%). It was noted that antihypertensive agents and antidiabetics should be interpreted differently in advanced cancer patients than in other patients, since higher values of blood pressure or blood glucose can be acceptable in this population, if asymptomatic.

\section{Preventative medications}

Todd et al. [15] carried out a systematic review into studies determining inappropriate use of preventive medication of 5 drug classes in patients with a life-limiting illness. In this review, they established a list with the most common inappropriate preventive medications used in this population (Table 1). Afterwards, they assessed the frequency of use of these medications in patients with advanced lung cancer in two hospitals (Table 2) [22]. No interventional study with the list of preventative medications has been published to date.

\section{Medications for chronic diseases}

Garfinkel et al. [16] described the medication use among end-stage cancer patients at the time of admission to home care hospice (Table 2). Medications that were included were used for chronic diseases. Drugs used for the oncological treatment were excluded. Appropriateness of medication was not assessed, but a stepwise recommendation for deprescribing was formulated based on the observation that at just 2 months before death, $23 \%$ of patients were still being treated with 12 or more drugs and $90 \%$ were still treated with 6 to 12 drugs.

\section{Tools non-specifically for cancer patients}

\section{Beers criteria}

The Beers criteria is a frequently used method to deprescribe in the overall geriatric population. Since the first edition, it has been updated many times based on new insights and evidence [17]. The Beers criteria are commonly applied on the palliative cancer population although it has been developed for the geriatric population. Zhou et al. [29] used the Beers criteria to estimate the frequency of six specific classes of PIMs in chemotherapy order templates for hematologic malignancies (Table 2). In $45 \%$ of these order templates, medications considered as PIMs by the Beers criteria were found. The authors wanted to draw attention to these potential risks. However, it could also be considered that the Beers criteria are not a perfect match to deprescribe in the cancer population. Karuturi et al. [23] applied the Beers criteria combined with drugs to avoid in the elderly (DAE) to identify PIM use in a cohort of older patients with stage II/III breast and colorectal cancer (Table 2). Also the frequency of these PIMs was evaluated at different time points. The use of PIM was lower at 3-6 months following initiation of chemotherapy when compared to baseline. Hong et al. [25] used the Beers criteria of 2015 to assess the frequency of PIMs in a population of geriatric patients with cancer undergoing first-line palliative chemotherapy (Table 2). However, medications typically used during chemotherapy (e.g., medications for nausea) were excluded. The authors state that modifying of the Beers criteria can be needed for the cancer population since several supportive drugs used during chemotherapy are considered PIMs by the Beers criteria. Nightingale et al. [26] combined 3 deprescribing guidelines: STOPP criteria, DAE, and the Beers criteria. The authors evaluated the frequency of PIMs in a population of ambulatory older adults with cancer. They divided the population based on the use of complementary and alternative medication (CAM). Herbal medications, minerals, or other dietary supplements, excluding vitamins, were considered as CAMs. The prevalence of the use of CAMs was $26.5 \%$. In 2017, the same research group assessed appropriateness of medication use by the Beers criteria in patients who received a comprehensive geriatric oncology assessment and received a pharmacist-led individualized medication assessment and planning (iMAP) intervention [28]. They enrolled 


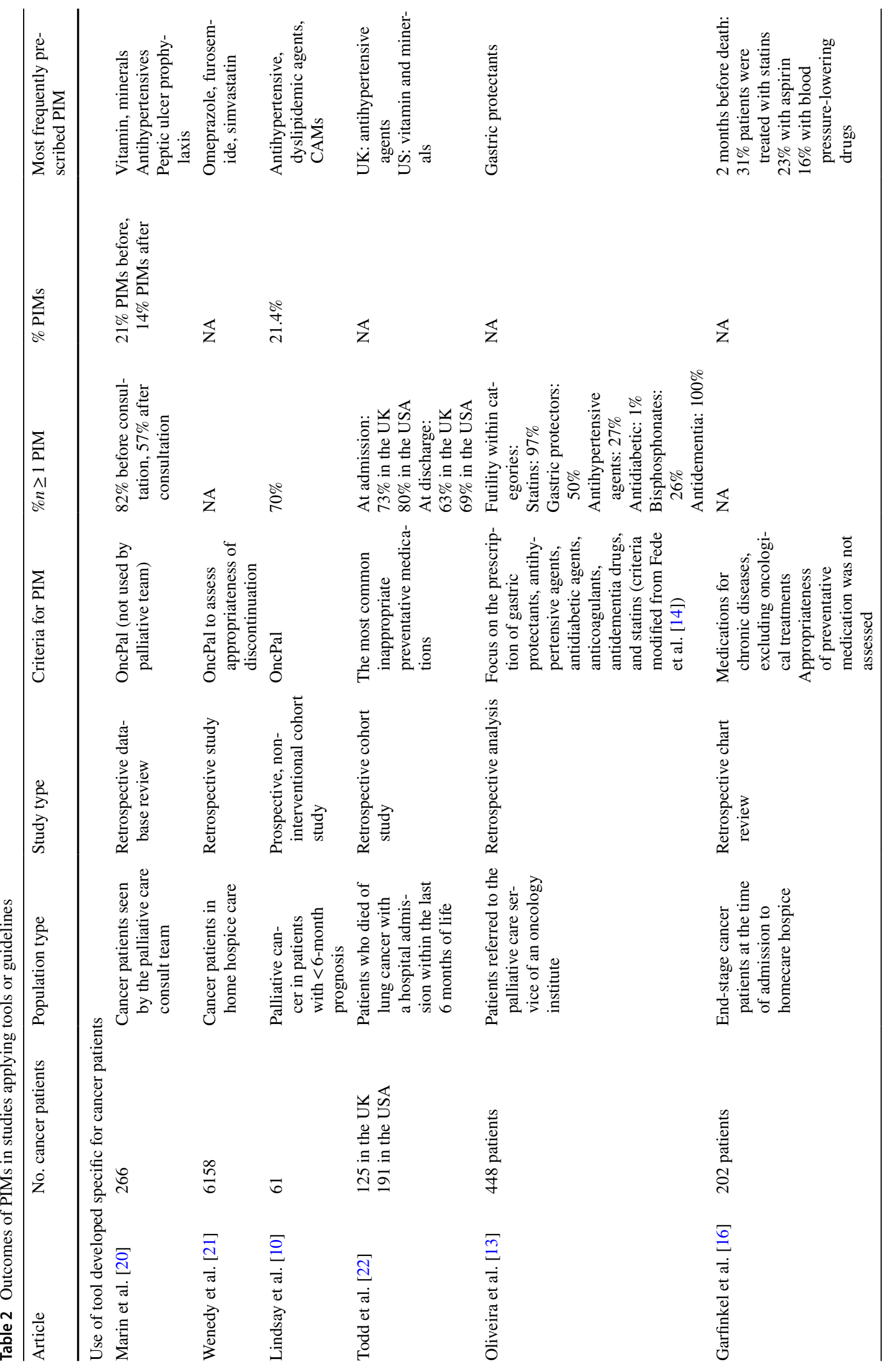




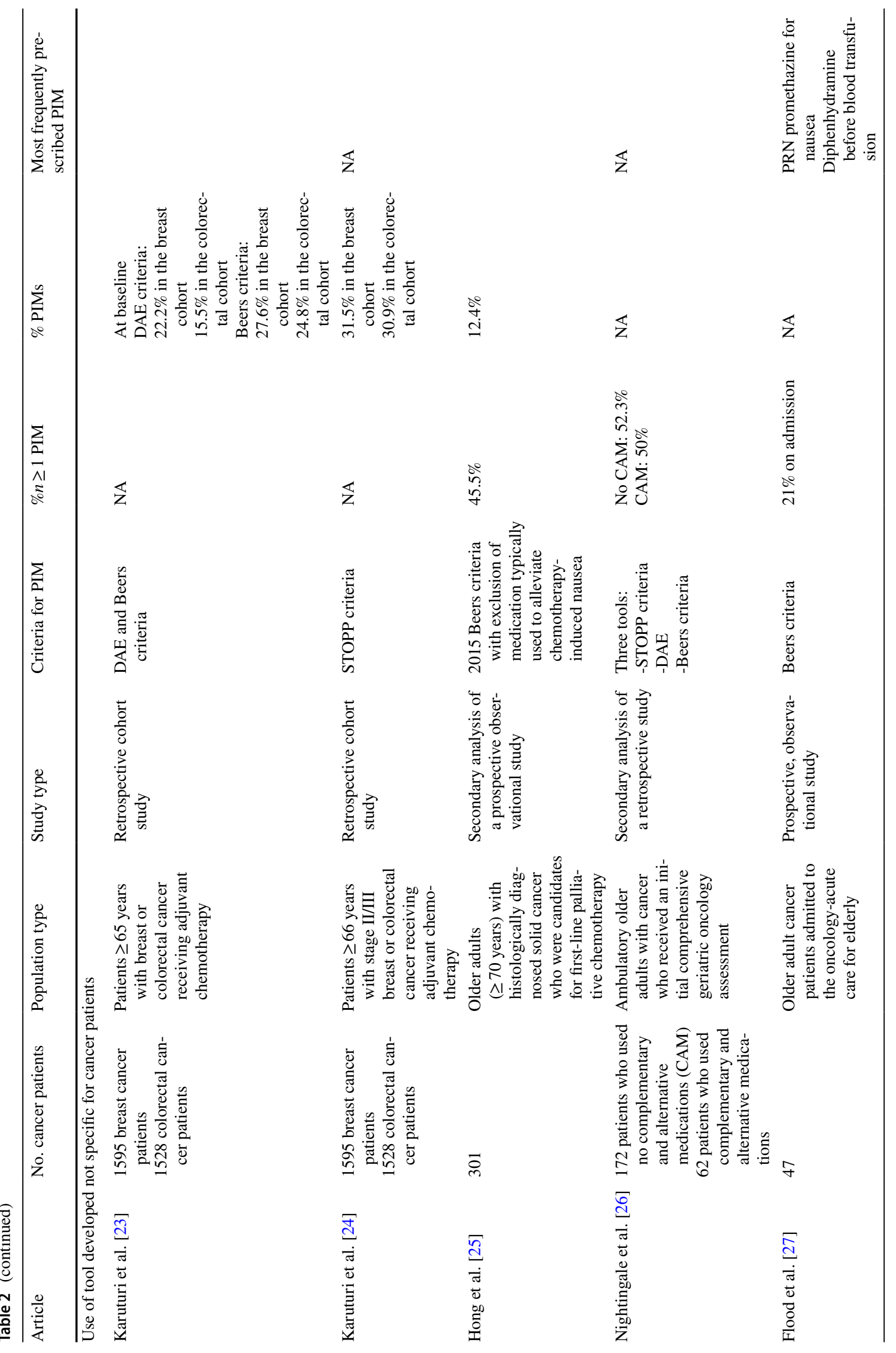




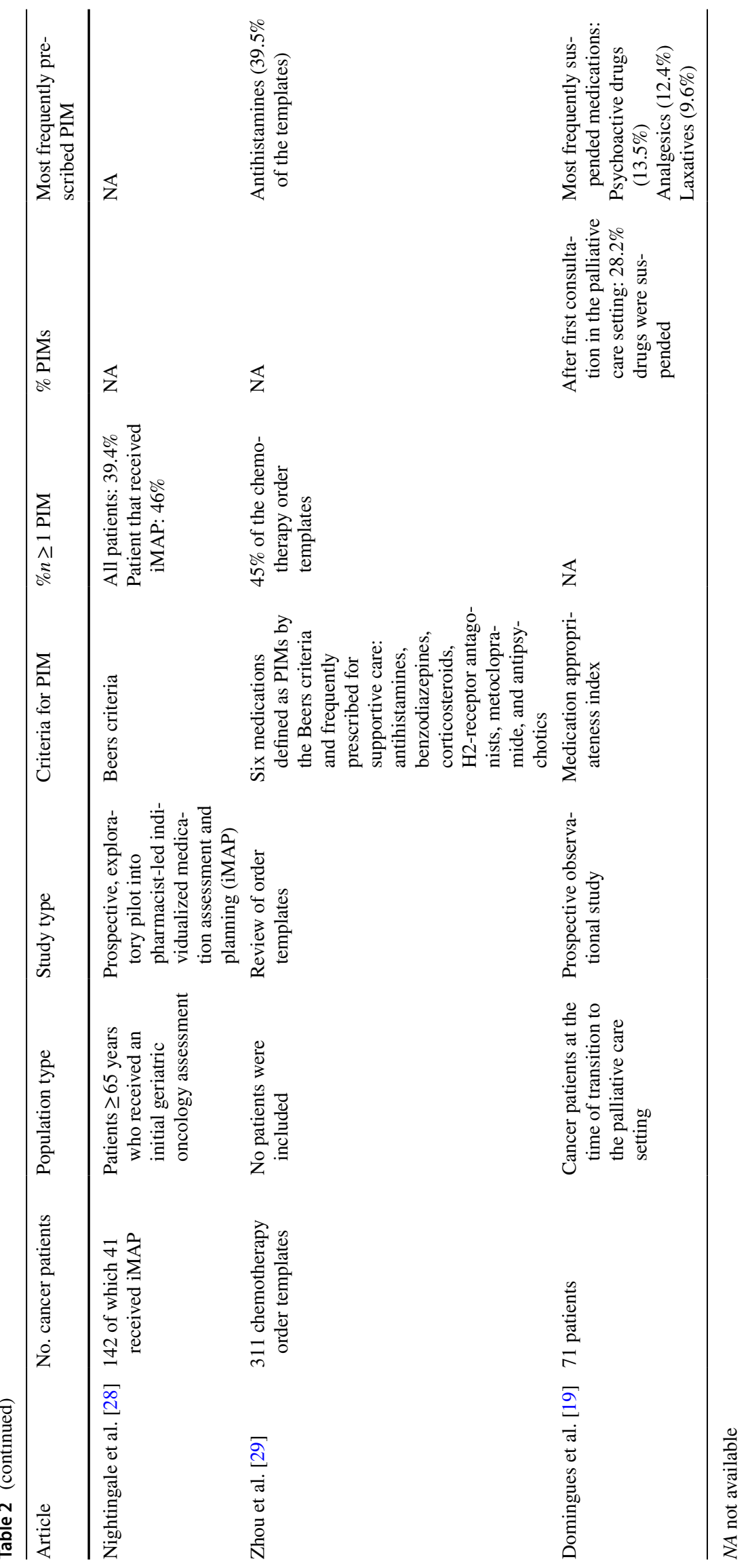


41 patients in their study and identified medication-related problems in $95 \%$. The pharmacists' interventions reduced the number of medication-related problems by $45.5 \%$. Flood et al. [27] finally applied the Beers criteria to 47 hospitalized older adult cancer patients referred to the acute care for elders unit (Table 1). The frequency of PIMs was determined on admission, and recommendations for deprescribing were made in $28 \%$ of patients. Again, the most frequent PIMs identified according to the Beers criteria were in fact drugs used as supportive care during cancer treatment.

\section{STOPP criteria}

STOPP criteria have been shown to improve inappropriate medication use in the elderly when applied during hospitalization [18]. Inappropriate medication use is associated with the occurrence of adverse drug events (ADEs) and an intervention using the STOPP criteria can reduce ADEs in older hospitalized patients. Karuturi et al. [24] used these criteria for estimating the use of PIMs in patients with stage II/III breast and colorectal cancer receiving chemotherapy, but found no statistically significant associations by the number of PIMs and clinical outcomes. As described above, Nightingale et al. [26] also used the STOPP criteria combined with 2 other guidelines.

\section{Medication appropriateness index}

The medication appropriateness index (MAI) is a questionnaire to assess futility of the drugs used (Table 1). It can be used to determine why the discontinued drug was inappropriate. Domingues et al. [19] applied a modified version of MAI for cancer patients at the time of palliative care transition in a prospective study (Table 2). They included 71 patients and found polypharmacy in $85 \%$ of cases. Using the MAI, 28\% of drugs used could be suspended.

\section{Discussion}

In this review, six deprescribing tools or guidelines specifically for cancer patients and three deprescribing tools or guidelines not specifically designed for cancer patients were identified.

On average, far more literature on the incidence of PIMs is available, than on methods for reducing them. This has also been noted in a large Delphi study evaluating better drug use in advanced disease [30]. The potential positive effects of deprescribing are multifold and have been demonstrated in several independent studies [2, 5-9]. Hence, to implement the concept of deprescribing in routine clinical care of palliative cancer patients, guidelines or consensus statements will be of great value.
Of the deprescribing tools we found for cancer patients, only the OncPal tool has been externally validated and has been applied by more than one research group [10, 20, 21]. The OncPal tool gives guidance for the majority of preventative drugs used, but lacks guidance on drugs used for thrombosis prophylaxis. In addition, no guidance on which drugs can be stopped in one step and which drugs should be tapered is given. Furthermore, OncPal has not been validated for palliative cancer patients with a life expectancy of more than 6 months whereas this population could also benefit from deprescribing. Of the deprescribing tools for the general population, the Beers criteria for geriatric patients have been studied most extensively and have also proven to be of value in cancer patients. Moreover, they are often updated and reviewed. However, some of the drugs that Beers criteria identify as being stoppable may actually be warranted in the specific group of cancer patients, either because they are part of supportive regimens for palliative chemotherapy, or because the risk-benefit is different in a palliative cancer patient when compared to a non-cancer geriatric patient. An example of the latter is the use of benzodiazepines to treat anxiety, which can be considered suboptimal due to tolerance, dependence, and fall-risk issues in general, but which in fact may be a good option if the expected end-of-life is too near to install other anxiety-reducing strategies.

Overall, we consider the OncPal guideline a good option and would recommend adding more drug classes, such as anticoagulants, to the tool. This population is at higher risk for deep vein thrombosis (DVT) due to their cancer or comorbidities, such as atrial fibrillation, and they are therefore more likely to use anticoagulants [31, 32]. However, limited clinical evidence is available regarding the prolonged use of anticoagulants as prophylaxis for palliative cancer patients. Furthermore, the frequently used anticoagulants coumarins can be a burden due to the regularly need of taking blood for INR monitoring. Unfortunately, some tumor types are associated with an increased risk of bleeding complications and increased risk for DVT [32]. Therefore, for each individual patient, the risk-benefit ratio should be assessed considering the use of anticoagulants. The OncPal guideline could well be combined with the stepwise methods described by Gonçalves [11] and Sharma et al. [12], because these methods incorporate a patient individualized approach. Finally, guidance from the Beers criteria on how to deprescribe (via stopping or via tapering) could well be a valuable addition to OncPal.

Future studies are needed to study the difference in clinical outcomes and quality of life in the palliative cancer population of the tools and guidelines identified in this review.

In conclusion, the tools, guidelines, and recommendations compiled in this review can help to support overdue standardization efforts to safely and effectively minimize unnecessary polypharmacy in palliative cancer patients. 
Author contribution Conceptualization: MC; literature search and data analysis: LM, MC; writing — original draft preparation: LM; writingreview and editing: MC.

Data availability N/A.

Code availability N/A.

\section{Declarations}

Ethics approval N/A.

Consent to participate N/A.

Consent for publication N/A.

Conflict of interest The authors declare no competing interests.

Open Access This article is licensed under a Creative Commons Attribution 4.0 International License, which permits use, sharing, adaptation, distribution and reproduction in any medium or format, as long as you give appropriate credit to the original author(s) and the source, provide a link to the Creative Commons licence, and indicate if changes were made. The images or other third party material in this article are included in the article's Creative Commons licence, unless indicated otherwise in a credit line to the material. If material is not included in the article's Creative Commons licence and your intended use is not permitted by statutory regulation or exceeds the permitted use, you will need to obtain permission directly from the copyright holder. To view a copy of this licence, visit http://creativecommons.org/licenses/by/4.0/.

\section{References}

1. Scott IA, Hilmer SN, Reeve E et al (2015) Reducing inappropriate polypharmacy: the process of deprescribing. JAMA Intern Med 175:827-834

2. Schenker Y, Park SY, Jeong K et al (2019) Associations between polypharmacy, symptom burden, and quality of life in patients with advanced, life-limiting illness. J Gen Intern Med 34:559566. https://doi.org/10.1007/s11606-019-04837-7

3. Morin L, Todd A, Barclay S et al (2019) Preventive drugs in the last year of life of older adults with cancer: is there room for deprescribing? Cancer 125:2309-2317. https://doi.org/10.1002/ cncr.32044

4. Lindsay J, Dooley M, Martin J et al (2014) Reducing potentially inappropriate medications in palliative cancer patients: evidence to support deprescribing approaches. Support Care Cancer 22:1113-1119. https://doi.org/10.1007/s00520-013-2098-7

5. Kutner JS, Blatchford PJ, Taylor DH et al (2015) Safety and benefit of discontinuing statin therapy in the setting of advanced, life-limiting illness a randomized clinical trial. JAMA Intern Med 175:691-700. https://doi.org/10.1001/jamainternmed.2015.0289

6. Garfinkel D (2018) Poly-de-prescribing to treat polypharmacy: efficacy and safety. Ther Adv Drug Saf 9:25-43. https://doi.org/ 10.1177/2042098617736192

7. Turner JP, Shakib S, Singhal N et al (2014) Prevalence and factors associated with polypharmacy in older people with cancer. Support Care Cancer 22:1727-1734. https://doi.org/10.1007/ s00520-014-2171-x

8. Cahir C, Fahey T, Teeling M et al (2010) Potentially inappropriate prescribing and cost outcomes for older people: a national population study. Br J Clin Pharmacol 69:543-552. https://doi. org/10.1111/j.1365-2125.2010.03628.x

9. Fralick M, Bartsch E, Ritchie CS, Sacks CA (2020) Estimating the use of potentially inappropriate medications among older adults in the United States. J Am Geriatr Soc 68:2927-2930. https://doi. org/10.1111/jgs. 16779

10. Lindsay J, Dooley M, Martin J et al (2015) The development and evaluation of an oncological palliative care deprescribing guideline: the 'OncPal deprescribing guideline.' Support Care Cancer 23:71-78. https://doi.org/10.1007/s00520-014-2322-0

11. Gonçalves F (2018) Deprescription in advanced cancer patients Pharmacy 6:88. https://doi.org/10.3390/pharmacy6030088

12. Sharma M, Loh KP, Nightingale G et al (2016) Polypharmacy and potentially inappropriate medication use in geriatric oncology. $\mathrm{J}$ Geriatr Oncol 7:346-353

13. Oliveira L, Ferreira MO, Rola A et al (2016) Deprescription in advanced cancer patients referred to palliative care. J Pain Palliat Care Pharmacother 30:201-205. https://doi.org/10.1080/15360 288.2016.1204411

14. Fede A, Miranda M, Antonangelo D et al (2011) Use of unnecessary medications by patients with advanced cancer: cross-sectional survey. Support Care Cancer 19:1313-1318. https://doi.org/ 10.1007/s00520-010-0947-1

15. Todd A, Husband A, Andrew I et al (2017) Inappropriate prescribing of preventative medication in patients with life-limiting illness: a systematic review. BMJ Support Palliat Care 7:113-121

16. Garfinkel D, Ilin N, Waller A et al (2018) Inappropriate medication use and polypharmacy in end-stage cancer patients: isn't it the family doctor's role to de-prescribe much earlier? Int J Clin Pract 72:1-7. https://doi.org/10.1111/ijcp.13061

17. Fick DM, Semla TP, Steinman M et al (2019) American Geriatrics Society 2019 updated AGS Beers Criteria ${ }^{\circledR}$ for potentially inappropriate medication use in older adults. J Am Geriatr Soc 67:674-694. https://doi.org/10.1111/jgs.15767

18. O'mahony D, O'sullivan D, Byrne S, et al (2015) STOPP/START criteria for potentially inappropriate prescribing in older people: version 2. Age Ageing 44:213-218. https://doi.org/10.1093/ageing/afu145

19. Domingues PGD, Carneiro R, Costa I et al (2015) Therapeutic futility in cancer patients at the time of palliative care transition: an analysis with a modified version of the medication appropriateness index. Palliat Med 29:643-651. https://doi.org/10.1177/ 0269216315573687

20. Marin H, Mayo P, Thai V et al (2020) The impact of palliative care consults on deprescribing in palliative cancer patients. Support Care Cancer 28:4107-4113. https://doi.org/10.1007/ s00520-019-05234-w

21. Wenedy A, Lim YQ, Lin Ronggui CK et al (2019) A study of medication use of cancer and non-cancer patients in home hospice care in Singapore: a retrospective study from 2011 to 2015. J Palliat Med 22:1243-1251. https://doi.org/10.1089/jpm.2018.0559

22. Todd A, Al-Khafaji J, Akhter N et al (2018) Missed opportunities: unnecessary medicine use in patients with lung cancer at the end of life - an international cohort study. Br J Clin Pharmacol 84:2802-2810. https://doi.org/10.1111/bcp.13735

23. Karuturi MS, Holmes HM, Lei X et al (2018) Potentially inappropriate medication use in older patients with breast and colorectal cancer. Cancer 124:3000-3007. https://doi.org/10.1002/cncr. 31403

24. Karuturi MS, Holmes HM, Lei X et al (2019) Potentially inappropriate medications defined by STOPP criteria in older patients with breast and colorectal cancer. J Geriatr Oncol 10:705-708. https://doi.org/10.1016/j.jgo.2019.01.024

25. Hong S, Lee JH, Chun EK et al (2020) Polypharmacy, inappropriate medication use, and drug interactions in older korean patients with cancer receiving first-line palliative chemotherapy. 
Oncologist 25:502-511. https://doi.org/10.1634/theoncologist. 2019-0085

26. Nightingale G, Hajjar E, Guo K et al (2015) A pharmacist-led medication assessment used to determine a more precise estimation of the prevalence of complementary and alternative medication (CAM) use among ambulatory senior adults with cancer. $\mathbf{J}$ Geriatr Oncol 6:411-417. https://doi.org/10.1016/j.jgo.2015.07. 003

27. Flood KL, Carroll MB, Le CV, Brown CJ (2009) Polypharmacy in hospitalized older adult cancer patients: experience from a prospective, observational study of an oncology-acute care for elders unit. Am J Geriatr Pharmacother 7:151-158. https://doi.org/10. 1016/j.amjopharm.2009.05.002

28. Nightingale G, Hajjar E, Pizzi LT et al (2017) Implementing a pharmacist-led, individualized medication assessment and planning (iMAP) intervention to reduce medication related problems among older adults with cancer. J Geriatr Oncol 8:296-302. https://doi.org/10.1016/j.jgo.2017.04.005
29. Zhou A, Holmes HM, Hurria A, Wildes TM (2018) An analysis of the inclusion of medications considered potentially inappropriate in older adults in chemotherapy templates for hematologic malignancies: one recommendation for all? Drugs Aging 35:459-465. https://doi.org/10.1007/s40266-018-0538-2

30. Huisman BAA, Geijteman ECT, Dees MK et al (2018) Better drug use in advanced disease: an international Delphi study. BMJ Support Palliat Care. https://doi.org/10.1136/bmjspcare-2018-001623

31. Kruger PC, Eikelboom JW, Douketis JD, Hankey GJ (2019) Deep vein thrombosis: update on diagnosis and management. Med J Aust 210:516-524. https://doi.org/10.5694/MJA2.50201

32. Gjam B, LF VD, FA K, MV H, (2018) Management and treatment of deep vein thrombosis in special populations. Expert Rev Hematol 11:685-695. https://doi.org/10.1080/17474086.2018.1502082

Publisher's note Springer Nature remains neutral with regard to jurisdictional claims in published maps and institutional affiliations. 